\title{
Hardware-in-the-loop evaluation of an LPV sliding mode fixed control allocation scheme on the MuPAL- $\alpha$ research aircraft
}

\author{
L. Chen ${ }^{1}$, H. Alwi ${ }^{1}$, C. Edwards ${ }^{1}$ and M. Sato ${ }^{2}$
}

\begin{abstract}
This paper develops a sliding mode fault tolerant control scheme based on an LPV system representation of the plant. The scheme involves a control allocation component, which is capable of fully utilizing the available actuators in the face of actuator faults. In this paper, information about the actuator faults is assumed not to be available and therefore a fixed control allocation structure is utilised in the event of faults. The proposed scheme is validated using the Japanese Aerospace Exploration Agency's Multi-Purpose Aviation Laboratory (MuPAL- $\alpha$ ) research aircraft. This paper describes initial hardware-in-the-loop (HIL) tests which serve as a precursor to upcoming real flight tests. The validation results show good lateral-directional state tracking performance in the fault free case with no visible performance degradation in the presence of (aileron) faults. Successful HIL tests demonstrate the potential of the proposed scheme which will be flight tested later this year.
\end{abstract}

\section{INTRODUCTION}

In the last decade, fault detection and diagnosis (FDD), and fault tolerant control (FTC) [1] have received significant attention from both the academic community and the aerospace industry. This is due to the fact that a large proportion of aircraft accidents during the last two decades were caused by loss of control, as a consequence of faults/failures occurring in the aircraft actuator and sensor systems. Furthermore, analytic redundancy FDD/FTC approaches can improve the sustainability of the aircraft, by breaking through the bottleneck of current hardware redundancy based fault detection systems. FTC systems can reduce the burden on the pilot's work load in the face of faults/failures without introducing an unnecessarily early direct control law reconfiguration. Some representative EU funded projects in this area include GARTEUR FM-AG16 [2], ADDSAFE [3], [4] and RECONFIGURE [5], [6]. These were devoted to narrowing the gap between advanced FDD/FTC approaches developed by the academic community and the practical and technical demands of industry.

The most recent investigation into the potential of FDD/FTC for aircraft flight control systems is the H2020/Japan cofunded project VISION (Validation of Integrated Safety-enhanced Intelligent flight CONtrol). The aim of the project is to develop and validate FDD/FTC techniques for improving aircraft Guidance, Navigation and Control. In the framework of VISION, recently proposed advanced FDD/FTC approaches are to be validated and evaluated at a system integration level in part using the Japanese Aerospace Exploration Agency's Multi-Purpose Aviation Laboratory (MuPAL- $\alpha$ ) research aircraft [7], [8].

In this paper, a sliding mode FTC scheme is developed and implemented within MuPAL- $\alpha$ 's Fly-By-Wire (FBW) system and then hardware-in-the-loop (HIL) tested. The proposed scheme combines the inherent robustness of sliding mode control (SMC) against uncertainty and faults/failures, and a fixed control allocation (CA) structure that fully utilizes the available redundancy within the system and redistributes the control signals to healthy actuators, without knowledge of the faults/failures. The scheme is developed within an linear parameter varying (LPV) framework. This allows FTC performance to be improved due to reduced design conservatism. Also the stability of the closed-loop scheme can be ensured over a wide range of flight conditions [9], [10], [11]. This paper focusses on lateral-directional control, and fault scenarios wherein the ailerons are assumed to operate at reduced effectiveness levels. The scheme does have the potential to cope with total actuator failure - but this is not pursued in this particular publication.

The objective of the paper is to develop FTC schemes that can retain close to nominal fault free performance despite actuator faults/failures. The main contributions of the paper is it describes the first application and validation of a sliding mode control allocation scheme at a system integration level where a real aircraft is involved in hardware-in-the-loop ground testing. The FTC scheme is different from the existing work on combining SMC with CA [12], [13] and exploits an LPV design framework.

The structure of the paper is as follows: Section II describes the LPV fault tolerant SMC scheme. The implemented control law, which is a combination of SMC and CA, is developed in the face of actuator faults/failures. In Section III, the effectiveness of the scheme is tested on Hardware-in-the-Loop (HIL) test platform of MuPAL- $\alpha$. The corresponding design and validation results are then presented. Section IV concludes the paper.

\footnotetext{
${ }^{1}$ College of Engineering, Mathematics and Physical Sciences, University of Exeter, EX4 4QF, UK. lc427@exeter.ac.uk, h.alwi@exeter.ac.uk, C.Edwards@exeter.ac.uk

2 Japan Aerospace Exploration Agency, Mitaka, Tokyo 181-0015, Japan. sato.masayuki@jaxa.jp
} 


\section{A. Problem formulation}

\section{LPV SLIDING MODE CONTROLLER DESIGN}

In this section, an LPV sliding mode FTC scheme with fixed CA is developed from a theoretical standpoint. Consider an LPV system with actuator faults/failures given by

$$
\dot{x}_{p}(t)=A_{p}(\rho) x_{p}(t)+B_{p}(\rho)\left(I_{m}-K(t)\right) u_{p}(t)
$$

where $A(\rho) \in \mathbb{R}^{n \times n}$ and $B(\rho) \in \mathbb{R}^{n \times m}$. The state vector and the control input are denoted by $x_{p} \in \mathbb{R}^{n}$ and $u_{p} \in \mathbb{R}^{m}$, respectively. The time varying scheduling parameter $\rho \in \mathbb{R}^{n_{r}}$ is assumed to be available and to belong to a compact set $\Omega \subset \mathbb{R}^{n_{r}}$. In this paper it is assumed $A(\rho)$ depends affinely on $\rho(t)$. In (1), the weighting matrix

$$
K(t):=\operatorname{diag}\left(k_{1}(t), \ldots, k_{m}(t)\right)
$$

where the $k_{i}(t) \in\left[\begin{array}{ll}0 & 1\end{array}\right]$, for $i=1 \ldots m$, are time varying scalars which represent the loss of effectiveness of the actuators [12]. For a fault-free actuator $k_{j}(t)=0$ and for a failed actuator $k_{j}(t)=1$. When $0<k_{j}(t)<1$, the actuator behaves with reduced effectiveness (i.e. a fault). Here it is assumed there are only $l$ controlled outputs:

$$
y_{c}(t)=C_{c} x_{p}(t)
$$

associated with (1) where $C_{c} \in \mathbb{R}^{l \times n}$ and $l \leq m$. Define integrator states according to

$$
\dot{x}_{r}(t)=r(t)-C_{c} x_{p}(t)
$$

where $r(t) \in \mathbb{R}^{l}$ represents the command signal which is differentiable and satisfies

$$
\dot{r}=\Gamma\left(r(t)-R_{c}\right)
$$

In (4), $R_{c}$ represents a fixed demand vector and $\Gamma \in \mathbb{R}^{l \times l}$ is a stable design matrix. The system in (4) represents a low-pass pre-filter and $\Gamma$ is a tuning parameter.

Define $x=\operatorname{col}\left(x_{r}, x_{p}\right)$ and create an augmented system

$$
\dot{x}(t)=\underbrace{\left[\begin{array}{cc}
0 & -C_{c} \\
0 & A_{p}(\rho)
\end{array}\right]}_{A(\rho)} x(t)+\underbrace{\left[\begin{array}{c}
0 \\
B_{p}(\rho)
\end{array}\right]}_{B(\rho)}(I-K(t)) u(t)+\underbrace{\left[\begin{array}{c}
I_{l} \\
0
\end{array}\right]}_{B_{c}} r(t)
$$

In (5) assume $B(\rho)$ can be factorized as

$$
B(\rho)=B_{v} B_{2}(\rho)
$$

where $B_{v} \in \mathbb{R}^{(n+l) \times l}$ is a fixed matrix, and $B_{2}(\rho) \in \mathbb{R}^{l \times m}$ is a matrix with varying components. For a discussions of the ramifications of this type of factorisation see [14]. This is also a feature of certain control allocation papers (in an LTI context) [15], [16].

Assumption 2.1: Assume $\operatorname{rank}\left(B_{2}(\rho)\right)=l$ for all $\rho \in \Omega$.

Assumption 2.2: The matrix pair $\left(A(\rho), B_{v}\right)$ is controllable and $\operatorname{rank}\left(B_{v}\right)=l$.

Since by assumption $\operatorname{rank}\left(B_{v}\right)=l$, there exists an orthogonal coordinate transformation which gives $B_{v}$ the partitioned form

$$
B_{v}=\left[\begin{array}{c}
0 \\
B_{v 2}
\end{array}\right]
$$

where $\operatorname{det}\left(B_{v 2}\right) \neq 0$. Then without loss of generality, after scaling the last $l$ states, the property $B_{v 2} B_{v 2}^{T}=I_{l}$ can always be achieved.

Then in particular (5) can be written as

$$
\dot{x}(t)=A(\rho) x(t)+\left[\begin{array}{c}
0 \\
B_{v 2} B_{2}(\rho)(I-K(t))
\end{array}\right] u(t)+B_{c} r(t)
$$

Define a virtual control signal $v(t) \in \mathbb{R}^{l}$ as

$$
v(t):=B_{v 2} B_{2}(\rho) u(t)
$$

If the actual physical control signals sent to the actuators are given by

$$
u(t):=B_{2}(\rho)^{\dagger} B_{v 2}^{T} v(t)
$$

where $B_{2}(\rho)^{\dagger}=B_{2}(\rho)^{T}\left(B_{2}(\rho) B_{2}(\rho)^{T}\right)^{-1}$ then equation (9) is satisfied. Consequently (8) can be written as

$$
\dot{x}(t)=A(\rho) x(t)+\left[\begin{array}{l}
0 \\
I
\end{array}\right] v-\left[\begin{array}{c}
0 \\
B_{v 2} B_{2}(\rho) K(t) B_{2}(\rho)^{\dagger} B_{v 2}^{T}
\end{array}\right] v(t)+B_{c} r(t)
$$

The design problem is to create a virtual control law $v(t)$ to ensure closed-loop stability of the system in (11) for all combination of faults/failures. In this paper a classic sliding mode controller will be designed [17], [18]. 


\section{B. Define of the switching function}

Partition the states in (11) as $x=\operatorname{col}\left(x_{1}, x_{2}\right)$ and define a switching function as

$$
s(t)=S x(t)
$$

where

$$
S=\left[\begin{array}{ll}
M & I_{l}
\end{array}\right]
$$

and $M \in \mathbb{R}^{l \times n}$ represents the design degree of freedom.

To analyse the performance of the closed-loop reduced order sliding motion as in [12], a coordinate transformation $\left(x_{1}, x_{2}\right) \mapsto$ $\left(x_{1}, s\right)$ is introduced. In this new coordinate system, equation (11) in partitioned form becomes

$$
\left[\begin{array}{c}
\dot{x}_{1}(t) \\
\dot{s}
\end{array}\right]=\left[\begin{array}{cc}
\hat{A}_{11}(\rho) & \hat{A}_{12}(\rho) \\
\hat{A}_{21}(\rho) & \hat{A}_{22}(\rho)
\end{array}\right]\left[\begin{array}{c}
x_{1}(t) \\
s
\end{array}\right]+\left[\begin{array}{l}
0 \\
I_{l}
\end{array}\right] v(t)-\left[\begin{array}{c}
0 \\
B_{v 2} B_{2}(\rho) K(t) B_{2}(\rho)^{\dagger} B_{v 2}^{T}
\end{array}\right] v(t)+\left[\begin{array}{c}
\hat{B}_{c 1} \\
\hat{B}_{c 2}
\end{array}\right] r(t)
$$

where

$$
\begin{aligned}
& \hat{A}_{11}(\rho):=A_{11}(\rho)-A_{12}(\rho) M \\
& \hat{A}_{12}(\rho):=A_{12}(\rho) \\
& \hat{A}_{21}(\rho):=M \hat{A}_{11}(\rho)+A_{21}(\rho)-A_{22}(\rho) M \\
& \hat{A}_{22}(\rho):=M A_{12}(\rho)+A_{22}(\rho)
\end{aligned}
$$

and the sub-blocks in (15) are obtained from partitioning the system matrix from (5) as

$$
A(\rho)=\left[\begin{array}{ll}
A_{11}(\rho) & A_{12}(\rho) \\
A_{21}(\rho) & A_{22}(\rho)
\end{array}\right]
$$

where $A_{11}(\rho) \in \mathbb{R}^{n \times n}$. During sliding $\dot{s}(t)=s(t)=0$, and the reduced order sliding motion is governed by

$$
\dot{x}_{1}(t)=\hat{A}_{11}(\rho) x_{1}(t)+\hat{B}_{c 1} r(t)
$$

Since $\hat{A}_{11}(\rho)$ is dependent on $M$ (see equation (15)) the choice of $M$ maybe viewed as a state feedback problem for the pair $\left(A_{11}(\rho), A_{12}(\rho)\right)$. Here $M$ will be selected to quadratically stabilize $\hat{A}_{11}(\rho)$. The MATLAB LMI multi-model state feedback synthesis code 'msfsyn' can be used to obtain the solution for the design matrix $M$.

\section{Control law with fixed control allocation}

In this section, a control law is selected to ensure and maintain a sliding motion [17], [18]. Here the virtual control law $v(t)$ is selected to contain two components: a 'linear' component $v_{l}$ and a 'nonlinear' component $v_{n}$; such that

$$
v=v_{l}+v_{n}
$$

Here specifically

$$
v_{l}=-\hat{A}_{21}(\rho) x_{1}(t)-\left(\hat{A}_{22}(\rho)-\Phi\right) s(t)-\hat{B}_{c 2} r(t)
$$

where $\Phi \in \mathbb{R}^{l \times l}$ is a stable design matrix and

$$
v_{n}=-\mathcal{K}(t, x) \frac{P_{2} s(t)}{\left\|P_{2} s(t)\right\|} \quad \text { if } \quad s(t) \neq 0
$$

where the symmetric positive definite matrix $P_{2} \in \mathbb{R}^{l \times l}$ satisfies

$$
P_{2} \Phi+\Phi^{T} P_{2}=-I_{l}
$$

For $K(t)$ as defined in (2) consider the inequality

$$
\left(B_{2}(\rho) K(t) B_{2}(\rho)^{T}\left(B_{2}(\rho)\right) K(t) B_{2}(\rho)^{T}\right)<(1-\epsilon)^{2}\left(B_{2}(\rho) B_{2}(\rho)^{T} B_{2}(\rho) B_{2}(\rho)^{T}\right)
$$

where $0<\epsilon \leq 1$. Define the admissible fault set

$$
\mathcal{F}_{\epsilon}=\{K(t) \text { in (2) such that inequality (22) is satisfied }\}
$$

Theorem 2.1: If the design matrix $M$ has been chosen such that $\hat{A}_{11}(\rho)$ is quadratically stable and $K(t) \in \mathcal{F}_{\epsilon}$, then choosing

$$
\mathcal{K}(t, x)>\frac{(1-\epsilon)\left\|v_{l}\right\|+\eta}{\epsilon}
$$

where $\eta$ is a positive design scalar, ensures a sliding motion takes place on $\mathcal{S}=\{x: S x=0\}$ in finite time. 


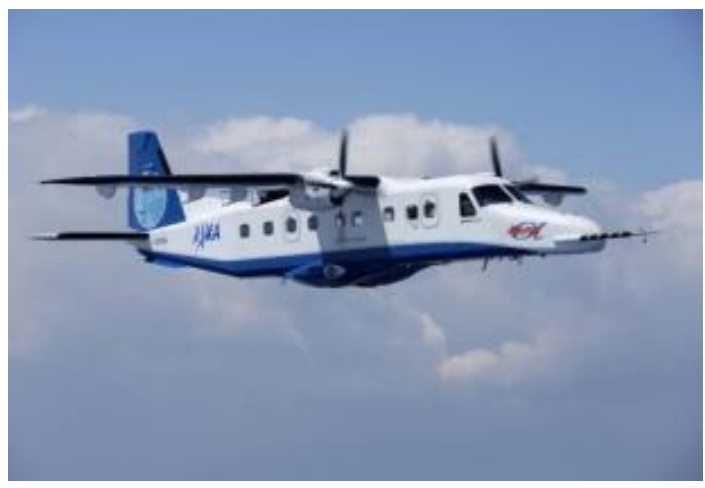

Fig. 1. MuPAL- $\alpha$ aircraft

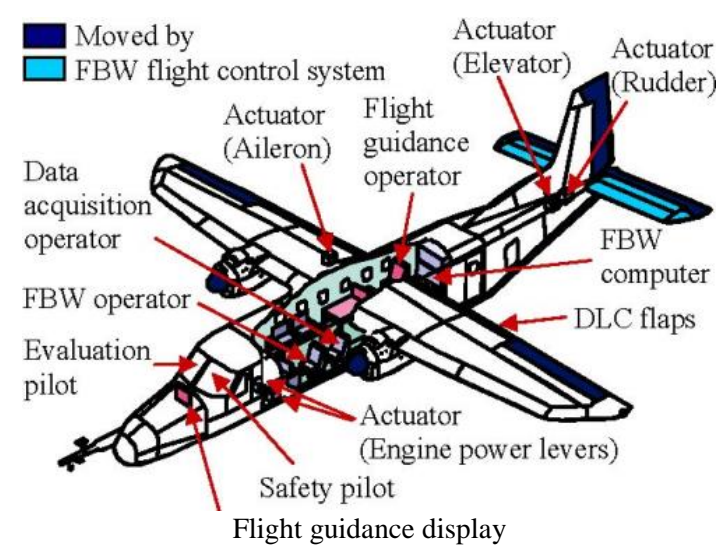

Fig. 2. Fly-by-wire configuration

Proof: due to space limitation, the proof is not included The actual input $u_{p}$ using fixed control allocation is

$$
u_{p}=B_{2}(\rho)^{T}\left(B_{2}(\rho) B_{2}(\rho)^{T}\right)^{-1} B_{v 2}^{T}\left(-\hat{A}_{21}(\rho) x_{1}(t)-\left(\hat{A}_{22}(\rho)-\Phi\right) s(t)-\hat{B}_{c 2} r(t)-\mathcal{K}(t, x) \frac{P_{2} s(t)}{\left\|P_{2} s(t)\right\|}\right)
$$

Note that (24) does not require knowledge of the faults $K(t)$.

\section{HIL TEST ON THE MUPAL- $\alpha$ PLATFORM}

\section{A. MuPAL- $\alpha$ platform}

MuPAL- $\alpha$ is a multi-purpose research aircraft used for testing advanced guidance and control technologies and evaluating research on human factors. It is a Dornier Do228-202 equipped with a research FBW system and Direct Lift Control (DLC) flaps to realize the variable stability and response capability. The MuPAL- $\alpha$ platform (see Fig. 1) supports both HIL tests and actual flight tests for advanced guidance and control technologies.

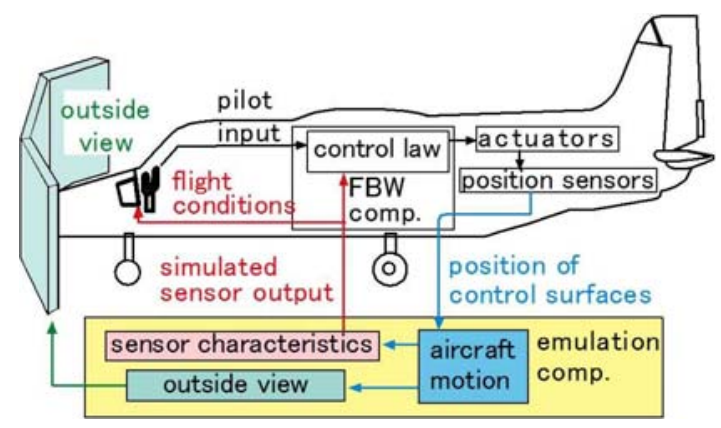

Fig. 3. HIL configuration 
Researchers are free to design motion control laws or the indicators for the pilot. This flexibility enables MuPAL- $\alpha$ not only to simulate the motion of various types of aircraft (big transports, business jets etc), but also to include gust responses tests and to investigate the effect of system failures (see for example [19], [8], [20], [21], [22]). It can be used to evaluate advanced control theories and newly developed navigation and guidance technologies. From a safety aspect, the primary elements of the FBW system are designed to be duplex, which makes it possible to detect system failure by cross-checking the signals. Whenever necessary, a safety pilot can override the FBW and take control through the Do228's mechanical control system [7] (see Fig. 2).

\section{B. MuPAL- $\alpha$ HIL test platform}

The HIL simulations were carried out to test the controller C-code implementation and to provide a valuable preliminary assessment of the controller performance before actual test flights. The tests were conducted for a wide range of manoeuvres and flight conditions under safe static conditions inside the hanger. The HIL test setup also has the capability to include various levels of wind and gust conditions to mimic actual flight conditions. The HIL tests allow the safety and evaluation pilots (in the cockpit, see Fig. 2) as well as the experimental operators (the FBW operator, data acquisition operator and flight guidance operator in the cabin, see Fig. 2) to familiarise themselves with the manoeuvres and procedures to be conducted during the actual flight tests.

In preparation for the test, the new control scheme has been written in C-code using a template provided by JAXA which contains the input-output interface with the rest of the FBW system.

Fig. 3 shows a schematic diagram of the configuration of the MuPAL- $\alpha$ HIL test platform. This configuration represents a typical flight simulator arrangement, but incorporates the actual aircraft as part of the setup. An emulation computer is responsible for providing the flight simulator capabilities and contains the high fidelity simulation model of the aircraft. The emulation computer takes the position of the actual aircraft control surfaces (which move during the HIL tests) as inputs to the simulation model. Based on the model the emulation computer then provides realistic 'sensor measurements' of the position and attitude of the aircraft. These measurements provide the signals to be used by the control law inside the FBW computer as well as providing flight conditions to the cockpit displays and panels. The other output of the emulation computer is the graphic display sent to a screen (which is placed on the outside of the cockpit) to provide pilots with the outside visualisation of the aircraft attitude. Once the HIL tests have been completed and the new control scheme has been 'burned' onto the FBW computer, the umbilical cords between the emulation computer and the FBW computer, are unplugged and removed from the aircraft, in preparation for the actual flight tests.

For further details of MuPAL- $\alpha$ and its operation, see for example [7].

\section{LPV modelling}

The LPV model of MuPAL- $\alpha$ was created via an interpolation of multiple linear time invariant (LTI) models [23] provided by Japanese Aerospace Exploration Agency (JAXA), which were derived under the following conditions:

- Altitude: $5000 \mathrm{ft}$ under standard atmosphere conditions

- Configurations: Flap and gear are set to up, DLC is at $0 \mathrm{deg}$, the weight of the aircraft is $5700 \mathrm{~kg}$, the center of gravity is at $28 \%$ and the engine speed is $100 \%$.

- Airspeed: The indicated airspeed ranges from $100 \mathrm{kts}$ to $200 \mathrm{kts}$.

Note that the LPV model has been established for modelling both longitudinal and lateral-directional dynamics. The scheduling parameters have been selected as

$$
\rho=\left[\begin{array}{ll}
v_{\text {ias }} & v_{\text {ias }}^{2}
\end{array}\right]
$$

where $v_{\text {ias }}$ represents the indicated airspeed. For the purpose of simplifying the LPV system synthesis and analysis, both scheduling parameters have been scaled to $\left[\begin{array}{ll}0 & 1\end{array}\right]$.

\section{Design results}

This paper will focuss on lateral-directional control (largely because it affords redundancy in terms of fault tolerant control). After reordering the lateral-directional states and removing the heading angle $\psi$, the system states are given by

$$
x_{p}=\left[\begin{array}{llll}
\phi & \beta & r & p
\end{array}\right]^{T}
$$

which denote roll angle, sideslip angle, yaw rate and roll rate, respectively. The system inputs $u_{p}$ are given by

$$
u_{p}=\left[\begin{array}{lll}
\delta_{t d} & \delta_{a} & \delta_{r}
\end{array}\right]^{T}
$$

where $\delta_{t d}$ represents differential trust, and $\delta_{a}$ and $\delta_{r}$ represent the aileron and rudder surface deflections. In this paper, differential trust is not used for control allocation. This is because the aircraft is on the ground during the HIL and it is not possible to use the engines to examine the effect of differential trust. 
The command signal is given by

$$
r=\left[\begin{array}{ll}
\beta_{c} & \phi_{c}
\end{array}\right]
$$

where $\beta_{c}$ and $\phi_{c}$ represent the sideslip angle and the roll angle commands respectively. In all the LTI models from different trim points (associated with the state order in (26)), the control derivatives are given by

$$
B_{p}=\left[\begin{array}{ccc}
0 & 0 & 0 \\
0 & Y_{\delta_{a}} / u_{0} & Y_{\delta_{r}} / u_{0} \\
\delta \bar{T}_{\delta_{t}} & N_{\delta_{a}} & N_{\delta_{r}} \\
\delta T_{\delta_{t}} & L_{\delta_{a}} & L_{\delta_{r}}
\end{array}\right]
$$

where $u_{0}$ represents the aircraft's constant (trimmed) forward velocity. During the controller synthesis, the control derivatives related to the $Y$ component were neglected since $Y_{\delta_{a}} / u_{0}$ and $Y_{\delta_{r}} / u_{0}$ are small (however the effect was evaluated in the HIL evaluations). The factorization of $B(\rho)$ (including the integrator states from (3) as the first two components) can be written as

$$
B(\rho)=B_{v} B_{2}(\rho)
$$

where

$$
B_{v}=\left[\begin{array}{ll}
0 & 0 \\
0 & 0 \\
0 & 0 \\
0 & 0 \\
\hline 1 & 0 \\
0 & 1
\end{array}\right] \quad \text { and } \quad B_{2}(\rho)=\left[\begin{array}{lll}
b_{51}(\rho) & b_{52}(\rho) & b_{53}(\rho) \\
b_{61}(\rho) & b_{62}(\rho) & b_{63}(\rho)
\end{array}\right]
$$

In what follows, using 'msfsyn' (a regional pole placement design [24], [25] to place the closed-loop poles to the left of the vertical line through -1 in the complex plane of the LMI region).

\section{E. Hardware-in-the-loop tests}

The results presented in the following subsections were obtained from Hardware-in-the-loop (HIL) tests. The proposed control scheme was initially coded in C. This code was integrated into the FBW system and compiled to generate the equipment code for the HIL test. During the HIL tests, the faults have been created at a software level. The signal out from the controller is modified in software to represent the fault and it is this modified signal which is sent to the actuators.

1) Fault free: The fault-free HIL test results are shown in Figs.4-6. The manoeuvre shown in this paper is manually created by the evaluation pilot via pedal and wheel manipulations. During the HIL test, a roll angle command from approximately $20 \mathrm{deg}$ to $-20 \mathrm{deg}$ is provided by the evaluation pilot, followed by a sideslip command from $-3 \mathrm{deg}$ to $3 \mathrm{deg}$. The trajectories of the lateral-directional states of the MuPAL- $\alpha$ are shown in Fig.4. The bottom plots from Fig.4 show good roll and sideslip tracking performance during fault free conditions. Figure 5 shows the corresponding sliding surfaces which demonstrates that sliding occurs despite the delay and uncertainty associated with the dynamics of the aileron and the rudder (details of the delay and the actuator dynamics can be found in [8]). The aileron and rudder commands and their surface deflections are shown in Fig. 6. It is clear from Fig. 6 that the aileron and the rudder are fault free and the actuator commands do not contain chattering. Note that for the fault free case, $K=\operatorname{diag}(1,0,0)$ since the differential trust is not used during the ground test.

2) Fixed CA: Figures 7-9 show the HIL test results when the aileron is faulty (i.e. only $70 \%$ efficiency). In this case, $K=\operatorname{diag}(1,0.3,0)$. A similar manoeuvre associated with the roll angle (20deg to $-20 \mathrm{deg}$ ) and sideslip angle ( $-3 \mathrm{deg}$ to $3 \mathrm{deg}$ ) is created by the evaluation pilot. The trajectories of the lateral-directional states of the MuPAL- $\alpha$ are shown in Fig. 7 and demonstrate the roll angle and the sideslip angle tracking performance can be maintained in the presence of the aileron fault. This demonstrates the FTC potential of the design. Furthermore the evaluation pilot did not feel the occurrence of the fault and unable to differentiate between fault-free and faulty conditions. The sliding surfaces are shown in Fig.8. The aileron and rudder commands and their surface deflections are shown in Fig. 9. The aileron deflection do not follow the demand due to the existence of the fault. It is clear from Fig. 9 the control law is acceptably smooth and does not possess visible chattering.

\section{CONCLUSION}

This paper has described the development and application of a sliding mode control FTC scheme for the Japanese Aerospace Exploration Agency's Multi-Purpose Aviation Laboratory (MuPAL- $\alpha$ ) research aircraft. The controller has been implemented on board the vehicle and integrated within the on-board FBW system. Hardware-in-the-loop tests have been undertaken whereby the vehicle remains on the ground but environment cues are provided to the pilot through vehicle models which interact and respond to the movement of the physical actuator. These tests are a requirement and a precursor to real flight tests, and validate the correct implementation of the controller and its interaction with the hardware. In this paper, a 

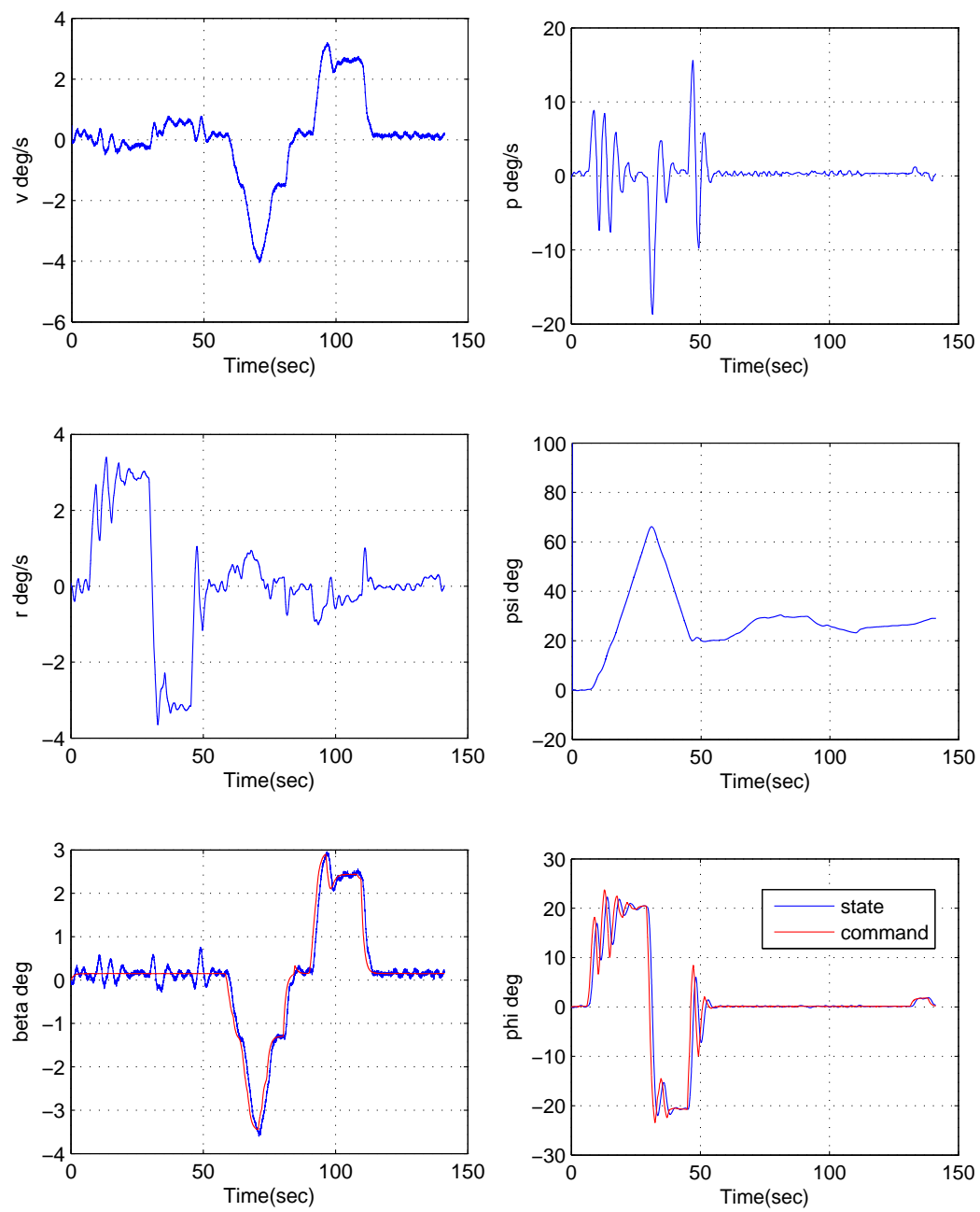

Fig. 4. Fault free states
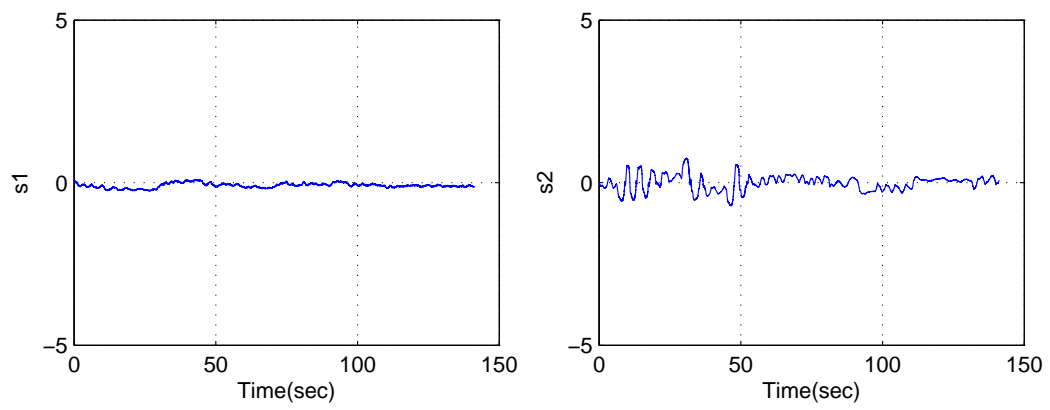

Fig. 5. Fault free sliding surfaces
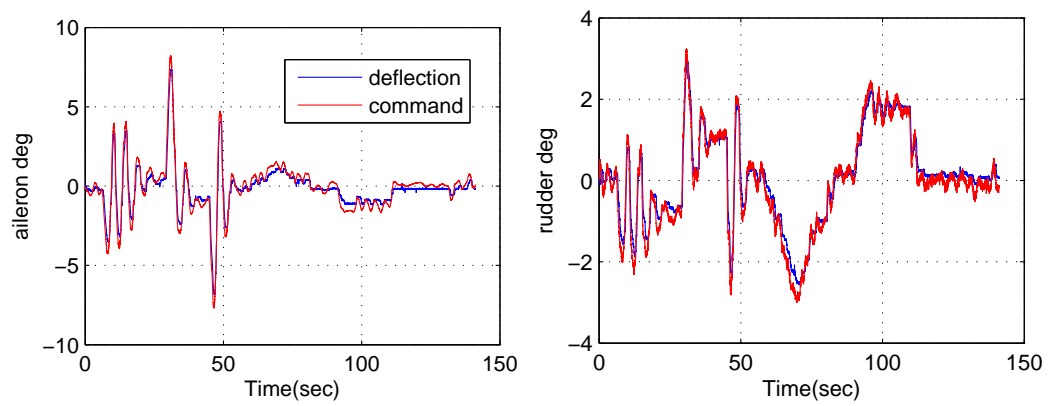

Fig. 6. Fault free control surface deflections 

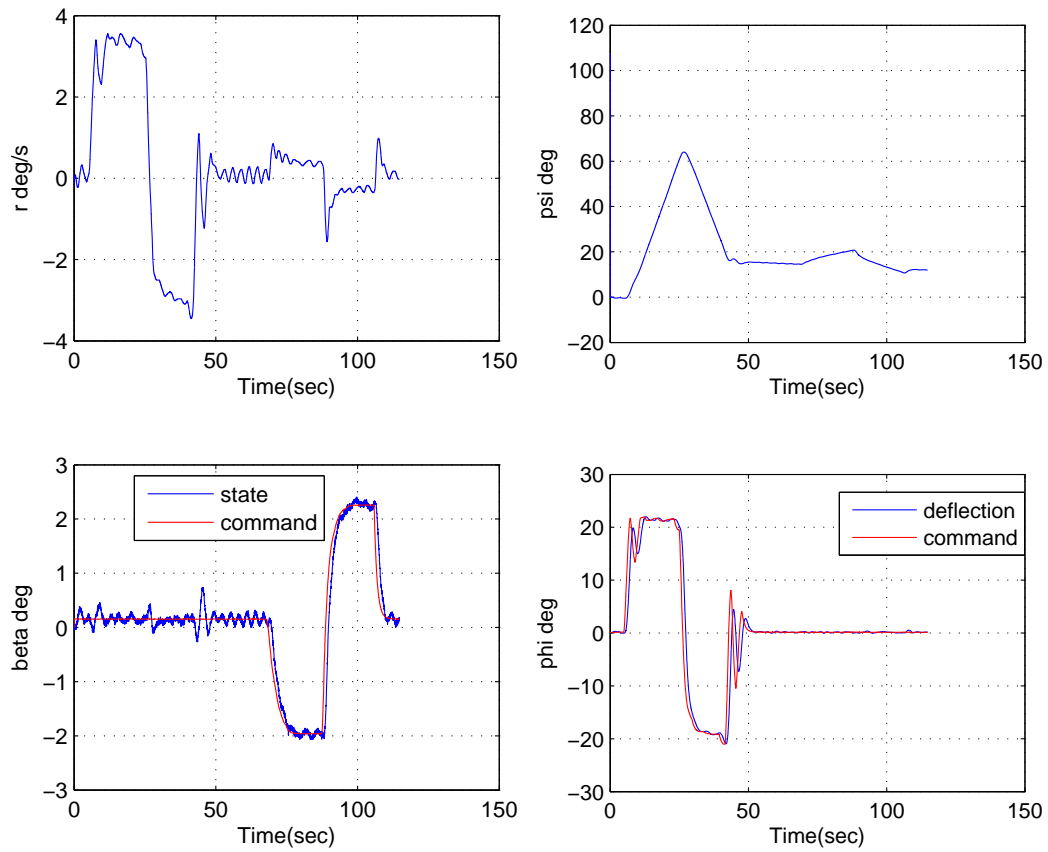

Fig. 7. States in the presence of the aileron fault
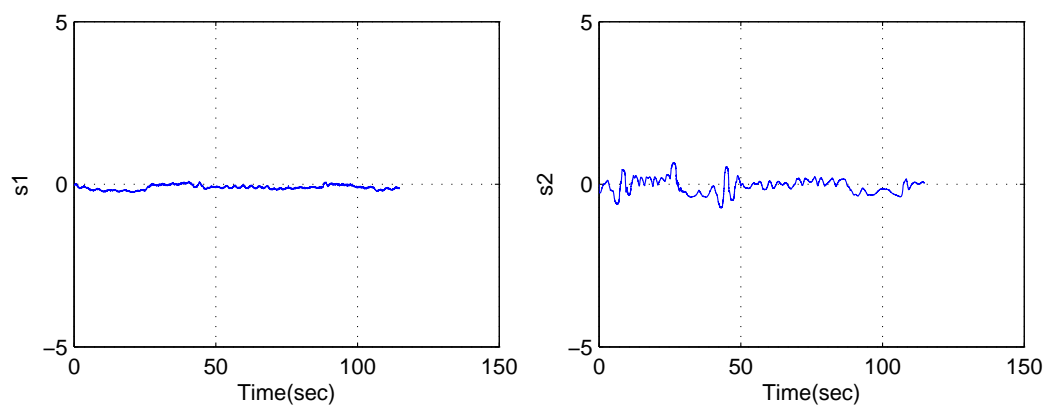

Fig. 8. Sliding surfaces in the presence of the aileron fault
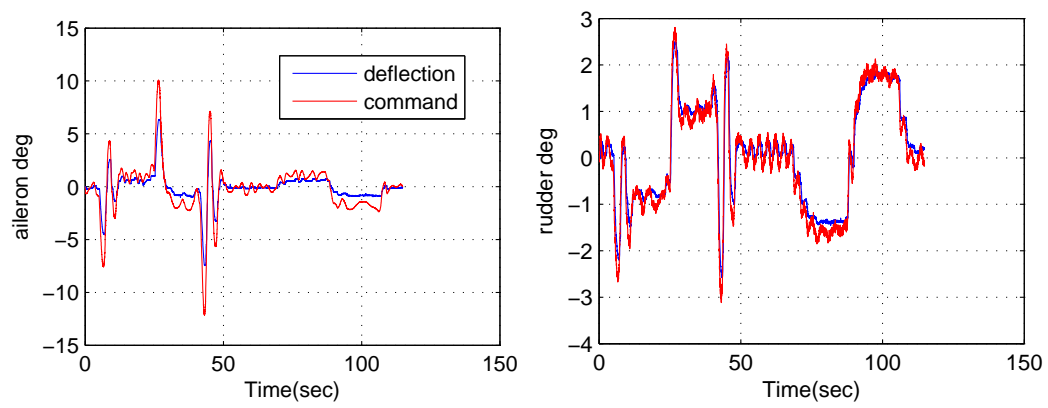

Fig. 9. Control surface deflections in the presence of the aileron fault 
fixed control allocation scheme is used to fully utilise the actuator availability in the face of actuator faults/failures, without real-time knowledge of the fault information. The Hardware-in-the-loop test results show that roll and sideslip tracking performance can be maintained in the presence of aileron faults, the actuator delay and uncertainties.

\section{ACKNOWLEDGEMENTS}

The research leading to these results has received funding from the European Union's Horizon 2020 research and innovation programme under grant agreement No.690811 and the Japan New Energy and Industrial Technology Development Organization under grant agreement No.062800, as a part of the EU/Japan joint research project entitled 'Validation of Integrated Safety-enhanced Intelligent flight cONtrol (VISION)'. The work presented here includes contributions from a number of people not directly involved with the preparation of the paper. In particular we gratefully acknowledge the contributions of T. Hosoya, M. Naruoka, J. Kawaguchi, S. Morokuma, H. Ishii and Y. Sagara from JAXA and Y. Uetake from Nakanihon Air Service for their support.

\section{REFERENCES}

[1] J. Chen and R. J. Patton, Robust model-based fault diagnosis for dynamic systems. Kluwer academic publishers, 1999.

[2] C. Edwards, T. Lombaerts, and H. Smaili, Fault tolerant flight control: A benchmark challenge. Springer, 2010.

[3] P. Goupil and A. Marcos, "Advanced diagnosis for sustainable flight guidance and control: The European ADDSAFE project," in SAE technical paper, 2011.

[4] - "The European ADDSAFE project: Industrial and academic efforts towards advanced fault diagnosis," Control Engineering Practice, vol. 31, pp. 109-125, 2014.

[5] P. Goupil, J. Boada-Bauxell, A. Marcos, P. Rosa, M. Kerr, and L. Dalbies, "An overview of the FP7 RECONFIGURE project: Industrial, scientific and technological objectives," in SAFEPROCESS '15, 2015.

[6] P. Goupil, J. Boada-Bauxell, A. Marcos, E. Cortet, M. Kerr, and H. Costa, "AIRBUS efforts toward advanced real-time fault diagnosis and fault tolerant control," in 19th IFAC World Congress, 2014.

[7] K. Masui and Y. Tsukano, "Developement of a new in-flight simulator MuPAL- $\alpha$," AIAA paper 2000-4574, Aug. 2000.

[8] M. Sato and A. Satoh, "Flight control experiment of multipurpose-aviation-laboratory- $\alpha$ in-flight simulator," Joural OF Guidance, Control and Dynamics, vol. 34, 2011.

[9] J. Mohammadpour and C. W. Scherer, Control of Linear Parameter Varying Systems with Applications. Springer, 2012.

[10] A. Maros and G. J. Balas, "Developement of linear parameter-varying models for aircraft," Journal of Guidance, Control and Dynamics, vol. 27, pp. 218-228, 2004.

[11] D. Rodonto, F. Nejjari, and V. Puig, "Robust quasi-LPV model reference FTC of a quadrotor UAV subject to actuator faults," International Journal of Applied Mathematics and Computer Sciences, vol. 25, pp. 7-22, 2015.

[12] H. Alwi, C. Edwards, and C. P. Tan, Fault Detection and Fault-Tolerant Control Using Sliding Modes. Springer, 2011.

[13] M. T. Hamayun, C. Edwards, and H. Alwi, Fault tolerant control schemes using integral sliding modes. Springer, 2016.

[14] H. Alwi and C. Edwards, "Fault tolerant control using sliding modes with on-line control allocation," Automatica, vol. 44, no. 7, pp. 1859-1866, 2008.

[15] O. Härkegård and S. T. Glad, "Resolving actuator redundancy - optimal control vs. control allocation," Automatica, vol. 41, no. 1, pp. 137-144, 2005.

[16] T. A. Johansen and T. I. Fossen, "Control allocation - a survey," Automatica, vol. 49, pp. 1087-1103, 2013.

[17] C. Edwards and S. K. Spurgeon, Sliding Mode Control: Theory and Applications. London, U.K.: Taylor \& Francis, 1998.

[18] Y. Shtessel, C. Edwards, L. Fridman, and A. Levant, Sliding Mode Control and Observation. Birkhauser, 2013.

[19] M. Sato and A. Satoh, "Simultaneous realization of handling and gust responses: In-flight simulator controller design," Joural OF Guidance, Control and Dynamics, vol. 31, 2008.

[20] S. Suzuki and A. Yanagida, "Research and development for fault tolerant flight control system part 1. intelligent flight control system," in 26th International Congress Of The Aeronautical Sciences, 2008.

[21] K. Masui, H. Tomita, and A. Yanagida, "Research and development for fault tolerant flight control system part 2 flight experiments," in 26th International Congress Of The Aeronautical Sciences, 2008.

[22] D. Tokunaga, K. Masui, and S. Suzukia, "Flight evaluation of fault-tolerant control system using simple adaptive control method," in Asia-Pacific International Symposium on Aerospace Technology, APISAT2014, 2014.

[23] H. Pfifer and S. Hecker, "Generation of optimal linear parametric models for LFT-based robust stability analysis and control design," IEEE Transactions on Control Systems Technology, vol. 19, pp. 118-131, 2011.

[24] M. Chilali and P. Gahinet, " $H_{\infty}$ design with pole placement constraints: An LMI approach," vol. 41, no. 3, 1996.

[25] P. Gahinet, A. Nemirovski, A. Laub, and M. Chilali, LMI Control Toolbox, User Guide. MathWorks, Inc., 1995. 